\title{
Cambios que Afectan el Clima Organizacional de una Empresa de Transporte
}

\section{Changes Affecting the Organizational Climate of a Transportation Company}

Manzanero Chan Miguel Ángel*, Sabido Domínguez Teresita de Jesús**

*Estudiante de Maestría en Gestión y Cambio Organizacional en la Facultad de Contaduría y Administración de la Universidad Autónoma de Yucatán. Email: manzaneromiguel@gmail.com, ORCID: https://orcid.org/0000-0002-8074-4258.

**Doctora en Administración. Facultad de Contaduría y Administración de la Universidad Autónoma de Yucatán. Email: tere.sabido@correo.uady.mx. ORCID: https://orcid.org/0000-0002-5424-1120.

Correo para recibir correspondencia: manzaneromiguel@gmail.com 
CAMBIOS QUE AFECTAN EL CLIMA ORGANIZACIONAL DE UNA EMPRESA DE TRANSPORTE.

\section{RESUMEN}

OBJETIVO: Señalar el cambio que sufre el clima organizacional y su repercusión en el cumplimiento de sus objetivos y en su operación de la organización, en un sindicato de trabajadores que migra al ámbito empresarial pero no cuenta con la asesoría adecuada de profesionales para realizar esos cambios legales, sociales y estructurales.

MATERIAL Y MÉTODO: Se utiliza el método cualitativo en este estudio, en específico el estudio de caso, con el cual se logra una descripción del panorama social antes y después de los cambios realizados. El material de investigación se obtuvo de entrevistas no estructuradas a los integrantes de la organización para conocer la percepción que tienen de sí mismos después de los cambios que implementó la organización.

RESULTADOS: Identificación de las causas y cambios que modificaron el clima organizacional y la percepción de sus integrantes después de estos cambios, es decir las consecuencias.

CONCLUSIONES: Cuando se llevan a cabo cambios organizacionales, sin la debida metodología que tome en cuenta las consecuencias de estos cambios en las personas se daña el clima organizacional y por ende se pone en riesgo la existencia misma de la organización, debido a que la inestabilidad organizacional es originada por la percepción de las personas después de los cambios, lo que culmina en la falta de colaboración para el logro de los objetivos de la empresa.

PALABRAS CLAVE: Clima Organizacional. Cambio Organizacional. Sindicato. Transporte.

\section{ABSTRACT}

OBJECTIVE: To point out the change that the organizational climate suffers and its repercussion on the fulfillment of the objectives and operation of the organization, in a union of workers that migrates to the business environment but does not have the adequate counseling from professionals to make those legal, social and structural changes. 
CAMBIOS QUE AFECTAN EL CLIMA ORGANIZACIONAL DE UNA EMPRESA DE TRANSPORTE.

MATERIAL AND METHOD: The qualitative method is used in this study, specifically the case study, with which a description of the social panorama is achieved before and after the made changes. The research material was obtained from unstructured interviews with the members of the organization to find out their perception of themselves after the changes implemented by the organization.

RESULTS: Identification of the causes and changes that modified the organizational climate and the perception of its members after these changes, that is, the consequences.

CONCLUSIONS: When organizational changes are carried out, without the proper methodology that takes into account the consequences of these changes on people, the organizational climate is damaged, and, therefore, the very existence of the organization is put at risk, because the organizational instability is originated by the perception of the people after the changes, which culminates in the lack of collaboration for the achievement of the objectives of the company.

KEY WORDS: Organizational Climate. Organizational Change. Labor union. Transport.

\section{INTRODUCCIÓN}

En la República Mexicana, durante las últimas cuatro décadas, se ha presentado un fenómeno de cambio en las organizaciones dedicadas a dar el servicio de transporte público de pasajeros; cambios observados y comentados por las personas que están inmersas en el gremio, como historias de vida, pero sin que sean documentadas en un artículo científico, que registre de forma adecuada el proceso en el que una organización de naturaleza sindical dedicada al transporte público se convierte en una empresa privada, y con ello los efectos que trae consigo la implementación de estos cambios en el comportamiento y percepción de sus integrantes.

En la publicación de artículos científicos se refieren al tema del transporte público, desde distintos puntos de vista, tal como lo hace Carmona (2016) que analiza el transporte desde el punto de vista del orden urbano, o como Pérez y Landín (2019) que lo hacen desde la perspectiva de la movilidad, aun así dentro de sus páginas, se puede encontrar el reconocimiento al trabajo de Rivera (2000) quien describe en una profunda investigación, la 
CAMBIOS QUE AFECTAN EL CLIMA ORGANIZACIONAL DE UNA EMPRESA DE TRANSPORTE.

configuración del transporte público en la Ciudad de México en la década de los noventas, así como las acciones que tomó el gobierno capitalino para obligar a los sindicatos existentes a que se convirtieran en una sola empresa privada, la cual nombraron Ruta 100 (p. 250-256).

Esta investigación de Rivera es lo más cercano a explicar de forma superficial, los problemas que tuvieron que enfrentar los integrantes de una o varias organizaciones sindicales que se fusionaron en una sola empresa privada, pero al no ser el objeto de estudio de su investigación, se convierte en un simple pasaje histórico de la evolución del transporte público en la capital mexicana.

En respuesta a la necesidad de documentar los cambios que se dan, en el interior de una organización de transporte, que migra de sindicato a empresa, y considerando primordialmente cómo afectan estos cambios a la conducta y percepción de los integrantes, es lo que da origen al presente documento.

¿Por qué es importante analizar el comportamiento y la percepción de los integrantes en una empresa? Molina, Briones, y Arteaga, (2016) consideran que el desarrollo de una empresa está basado en la gestión de la actitud de las personas que participan en ella, es decir, que el comportamiento organizacional define la eficiencia y la eficacia de las organizaciones, ya sean empresas, escuelas, organismos de gobierno u organizaciones de servicios entre otros (p. 500).

La importancia del comportamiento y la percepción de sus integrantes se acentúa cuando se trata de una organización que se basa en la representación colectiva de sus partes, tal es el caso de una organización sindical, ya que de origen su objetivo es la representación de las necesidades de quienes la conforman, siendo una parte primordial la identificación de las personas con la organización, la sana convivencia que exista entre sus integrantes, y el comportamiento que tiene en general la organización con su medio ambiente.

Es así, como lo plasma Schmalz (2017) cuando habla del poder asociativo que tienen las organizaciones sindicales, y que contempla que ese poder asociativo está basado en la coherencia interna, la cual permite la existencia de una identidad colectiva que posibilita la resolución de conflictos y la realización de proyectos en conjunto; siempre y cuando la organización demuestre una flexibilidad que le permita compaginar su estructura con los intereses de los afiliados para poder aprovechar su poder de asociación, que fue el motivo principal de su creación. 
CAMBIOS QUE AFECTAN EL CLIMA ORGANIZACIONAL DE UNA EMPRESA DE TRANSPORTE.

De modo que, estudiar el comportamiento de las personas dentro de una organización, o el sentido de pertinencia, o el impacto social, y las consecuencias en general en el desempeño de la organización, es estudiar el clima organizacional (Salazar, Guerrero, Machado y Cañedo, 2009, párr. 9-10).

De acuerdo con Galarza (2015) quien cita a Brunet, Silva y Campbell, el clima organizacional se clasifica con la ayuda de las siguientes dimensiones básicas: Autonomía individual: Que se refiere a la responsabilidad, independencia y poder de decisión que goza el sujeto en su organización. Grado de estructura del puesto: Que apunta a la forma en la que se establecen y transmiten los objetivos y métodos de trabajo al personal. Recompensa: Referido a los aspectos económicos y a las posibilidades de promoción. Consideración, agradecimiento y apoyo: Relativo a las formas en que el empleado recibe estímulos de sus superiores (p. 356-357).

Cada una de estas dimensiones básicas, ayudan a comprender la integración y participación de las personas que pertenecen a la empresa, siendo estos conceptos los que en conjunto le permiten al individuo desarrollarse dentro de su organización y establecer su posición, así como su colaboración en los resultados.

Por su parte, Domínguez, Ramírez y García (2013) aseguran que cuando los individuos se sienten bien identificados con la organización o cuando consideran que los objetivos de la empresa y sus objetivos individuales convergen, se genera una lealtad hacia la empresa llamada compromiso organizacional. Por el contrario, cuando no se establece o fomenta este compromiso organizacional se produce una disminución de la eficiencia y eficacia de la organización (p. 61).

Por lo tanto, se requiere conocer la percepción de los miembros de una organización para conocer si se experimentó un cambio positivo o negativo en el clima organizacional. Tratándose del presente caso de estudio, se propone la siguiente hipótesis: cuando una organización sindical de transporte público modifica su estructura para convertirse en una empresa privada, deteriora su clima organizacional.

En ese sentido, se establece como objetivo general del presente documento, analizar el cambio en el clima organizacional de la empresa. Para ello, se establece como objetivos específicos, conocer la percepción de las personas ante los cambios implementados y clasificar de acuerdo con la percepción de los integrantes, las dimensiones básicas que componen el clima organizacional. 
CAMBIOS QUE AFECTAN EL CLIMA ORGANIZACIONAL DE UNA EMPRESA DE TRANSPORTE.

\section{MATERIAL Y MÉTODO}

Para la realización del presente documento, se llevó a cabo una investigación cualitativa, utilizando entrevistas no estructuradas a los integrantes de la organización, y se suma la observación del investigador, quien se sumerge en el medio ambiente de la empresa para poder visualizar las afectaciones psicosociales presentes, tal y como lo menciona Hernández, Fernández y Batista (2010) la investigación cualitativa se basa en el proceso mismo de recolección y análisis, recordando que es interpretativa y que el investigador puede hacer su propia descripción y valoración de los datos (p. 370).

Se recolecta información en 3 líneas de acción:

- A través de entrevistas a miembros de la organización.

- Se realiza una investigación en medios digitales.

- La observación del investigador dentro de la empresa.

Se realizaron 30 entrevistas a socios de la organización que de acuerdo con su clasificación interna, tienen el puesto de:

- Socio Administrador.

- Socio Operador.

Las limitaciones que se deben considerar del estudio de caso se pueden definir de la siguiente manera:

- Al obtener la información de fuentes primarias, a través de las entrevistas a socios administradores y socios operadores, se observó temor en las personas al aportar los datos dentro de las instalaciones de la empresa.

- Considérese que la información obtenida y plasmada en este documento, se recabó antes de marzo del año 2020, fecha en la que se declaró la pandemia en los Estados Unidos Mexicanos por el SARS-CoV-2, mundialmente conocido como COVID-19.

- En el presente documento, se plasma en resumen los comentarios y platicas de los socios administradores y operadores que estuvieron dispuestos a realizar la entrevista, a diferencia de aquellos que declinaron realizarla por motivos personales, por lo que considere la diferencia que pudiera tenerse al obtener un mayor número de entrevistados, que forman el universo de los integrantes de toda la organización. 
CAMBIOS QUE AFECTAN EL CLIMA ORGANIZACIONAL DE UNA EMPRESA DE TRANSPORTE.

- Este documento se realiza con la información obtenida del caso de estudio, y debido a que no tiene antecedentes en el giro del transporte público en la república mexicana, se complementó la información con investigación periodística de fuentes digitales.

Para efectos de confidencialidad solicitada por la empresa, no se mencionará su nombre, ni datos personales, ni otros datos que pudieran descifrar la organización de la que se trata, la información será configurada para poder ser analizada pero no identificable en lo mayor posible, ya que se trata de una empresa que es icónica en la ciudad de Mérida, Yucatán, y muy fácil de identificar si no se tiene el cuidado con los datos recabados.

Para fines del presente documento y para conservar la confidencialidad de los datos obtenidos de la empresa, a solicitud de su representante, se le nombrará Agrupación de Transportistas de Yucatán (ATY).

El caso de estudio se trata de una empresa de transporte público de la ciudad de Mérida, Yucatán, que originalmente nació como sindicato, pero decidió implementar cambios, entre 2009 y 2019, que la transformaron en una empresa privada. En el año 2020, consta de 200 socios administradores y 800 socios operadores, así como más de 400 unidades o vehículos de transporte.

La organización tiene una configuración de empresa, por lo que cuenta con una asamblea general de socios, y con un comité directivo de 28 personas, de las cuales, uno es el presidente. La empresa ha realizado varios cambios en su estructura legal, administrativa y social, estos cambios implementados se recolectaron como resultado de las entrevistas realizadas a los socios administradores y operadores, y se presentan en tablas para su mejor comprensión.

Los cambios que implementó la empresa ATY para dejar de ser un sindicato, se clasifican en tres tipos: cambios legales, cambios en la estructura administrativa y cambios sociales.

\section{Cambios Legales}

Los cambios legales fueron los más significativos que tuvo la empresa ATY, debido al objetivo de conformarse en una empresa, encontraron en el cambio de razón social el motivo ideal para ir avanzando hacia su objetivo empresarial. 
CAMBIOS QUE AFECTAN EL CLIMA ORGANIZACIONAL DE UNA EMPRESA DE TRANSPORTE.

Al realizar el cambio de razón social, forzosamente tenía que cambiar el tipo de administración, puesto que la razón social implicaba estar sujeto a una diferente ley de acuerdo con la normatividad vigente en el estado de Yucatán, dado que debido a la ley que rige en su caso la situación civil o comercial, de cada organización, es imperante el nombramiento de nuevos puestos y funciones, así como la modificación en el patrimonio invertido.

En la Tabla 1, se simplifica e identifica cada cambio de razón social con su respectiva modificación en la situación legal y su tipo de administración.

\section{Cambios en la Estructura Administrativa}

Para cada cambio en su razón social, la empresa modificó la forma de su estructura de trabajo, de tal manera que, en cada cambio legal, modificó también la estructura administrativa con la que regía sus operaciones diarias, sometiendo a sus colaboradores a un continuo cambio de puestos, funciones y líneas de autoridad. En la Figura 1, se muestra la estructura administrativa con la que inicio la empresa, una organización sindical, conformada únicamente por operadores de transporte.

Tabla 1

Cambios Legales

\begin{tabular}{lll}
\hline Razón Social & \multicolumn{1}{c}{ Situación legal } & \multicolumn{1}{c}{ Tipo de administración } \\
\hline Sindicato obrero & $\begin{array}{l}\text { Regido por la Ley Federal } \\
\text { del Trabajo }\end{array}$ & $\begin{array}{l}\text { Trabajadores agremiados, representados } \\
\text { por un secretario general. }\end{array}$ \\
Asociación civil & $\begin{array}{l}\text { Regido por el Código Civil } \\
\text { del Estado de Yucatán }\end{array}$ & $\begin{array}{l}\text { Asociados representados por un } \\
\text { presidente y un comité ejecutivo. }\end{array}$ \\
Sociedad anónima & $\begin{array}{l}\text { Regido por el Código de } \\
\text { Comercio del Estado de un comité directivo. } \\
\text { Yucatán }\end{array}$ & $\begin{array}{l}\text { Socios representados por un presidente y } \\
\text { un }\end{array}$ \\
\hline
\end{tabular}

Fuente: Elaboración propia con datos de la empresa, febrero 2020. 


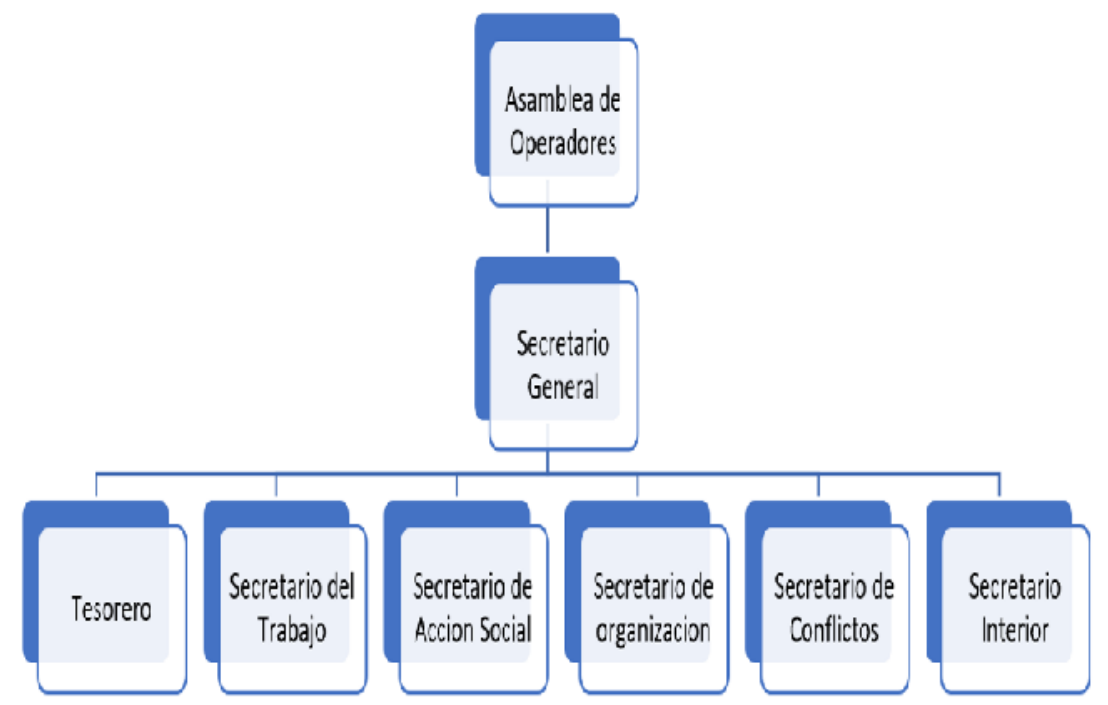

Figura 1. Estructura Administrativa como Sindicato Obrero.

Fuente: Elaboración propia con datos de la empresa, febrero 2020.

Cabe mencionar, que estas estructuras presentadas no representan todos los puestos y funciones reales, ya que la finalidad es exponer el número de niveles jerárquicos con los que inicio el sindicato, la representación que tenía y las líneas de autoridad que imperaba.

En la siguiente Figura, se puede observar el distanciamiento del representante respecto a sus representados, la estructura de asociación civil, siendo que los socios operadores aún se les considera parte de la asamblea general, pero ya no tienen una comunicación directa con su representante, debido a la creación de nuevos puestos y funciones.

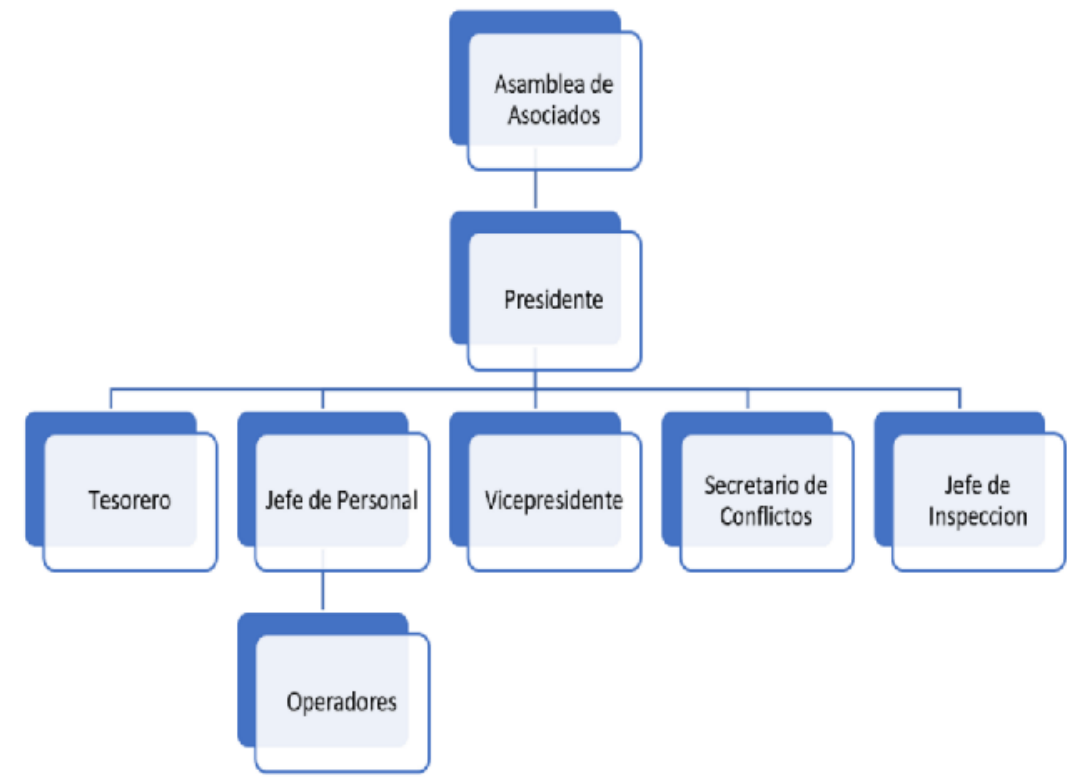

Figura 2. Estructura Administrativa como Asociación Civil.

Fuente: Elaboración propia con datos de la empresa, febrero 2020. 
CAMBIOS QUE AFECTAN EL CLIMA ORGANIZACIONAL DE UNA EMPRESA DE TRANSPORTE.

Por último, en la Figura 3, la estructura de empresa, en la que sus socios operadores terminan por separarse y alejarse de su representante, debido a que, en la asamblea de socios, solo se encuentran los socios administradores, siendo los privilegiados en obtener la categoría de socios capitalistas y los socios operadores se convierten en empleados.

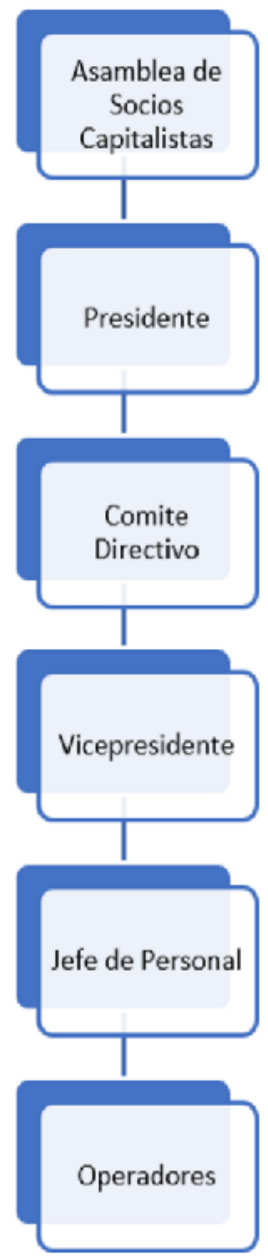

Figura 3. Estructura Administrativa Conformada como Sociedad Anónima.

Nota. Fuente: Elaboración Propia con datos de la empresa, febrero 2020.

\section{Cambios Sociales}

Después de haber sufrido cambios en su estructura legal y en su estructura de trabajo, esto llevo a que socialmente también se suscitarán cambios con respecto a la forma de percibir a sus agremiados (Tabla 2). 
CAMBIOS QUE AFECTAN EL CLIMA ORGANIZACIONAL DE UNA EMPRESA DE TRANSPORTE.

Tabla 2

Identidad del Agremiado en la Estructura Original del Sindicato

\begin{tabular}{ll}
\hline Categoría de Integrante & \multicolumn{1}{c}{ Derechos dentro de la Organización } \\
\hline \multirow{3}{*}{ Operador Agremiado } & - Todos tienen los mismos derechos. \\
& - Tiene voz y voto. \\
\hline
\end{tabular}

Fuente: Elaboración propia con datos de la empresa, febrero 2020.

Tabla 3

Identidad del Agremiado con la Estructura de Asociación Civil

Categoría de Integrante Derechos dentro de la Organización

\begin{tabular}{|c|c|}
\hline Socio C & $\begin{array}{l}\text { - } \quad \text { Identificado como operador. } \\
\text { - } \quad \text { Tiene voto, pero no voz. } \\
\text { - } \text { niene derecho a elegir a su representante, pero no puede ser } \\
\text { ninte ni parte del comité ejecutivo. }\end{array}$ \\
\hline Socio B & $\begin{array}{l}\text { - Es el socio C que acumulo } 10 \text { años de antigüedad, ya puede } \\
\text { adquirir su propio vehículo, cuando así lo permita el } \\
\text { crecimiento de la organización. } \\
\text { - Tiene voto, pero no tiene voz. } \\
\text { - Puede elegir a su representante, pero no puede ser ni } \\
\text { representante ni parte del comité ejecutivo. }\end{array}$ \\
\hline Socio A & $\begin{array}{l}\text { - Ya administra su propio vehículo. } \\
\text { - } \text { Tiene voz y voto. } \\
\text { - Puede elegir a su representante. } \\
\text { Puede ser elegido representante o ser parte del comité } \\
\text { ejecutivo. }\end{array}$ \\
\hline
\end{tabular}

Fuente: Elaboración propia con datos de la empresa, febrero 2020.

Tabla 4

Identidad del Agremiado en la Actual Estructura de Sociedad Anónima

\begin{tabular}{ll}
\hline Categoría de Integrante & \multicolumn{1}{c}{ Derechos dentro de la Organización } \\
\hline \multirow{2}{*}{ Socio } & Es el socio A qué se convirtió en socio capitalista. \\
& - Tiene voz y voto. \\
& Tiene derecho a elegir a su representante. \\
& directivo. \\
Operador & Es el socio B o C que al no tener vehículo propio se le \\
& considera únicamente empleado. \\
& No tiene voz y no tiene voto. \\
& No tiene derecho a elegir ni a ser elegido representante ni \\
& parte del comité directivo. \\
\hline
\end{tabular}

Fuente: Elaboración propia con datos de la empresa, febrero 2020. 
CAMBIOS QUE AFECTAN EL CLIMA ORGANIZACIONAL DE UNA EMPRESA DE TRANSPORTE.

Con respecto a la percepción que tienen los socios administradores y operadores de esta organización, se tiene como resultado de las entrevistas no estructuradas, una serie de respuestas que se dieron de acuerdo con el sentimiento de pertenencia de cada uno de ellos.

Sin embargo, fue necesario dividirlo en dos tipos de respuesta: la percepción del socio administrador quien hoy es considerado el socio capitalista y las respuestas de los socios operadores quienes hoy en día se les considera como operadores-empleados. Al mismo tiempo, se concentraron las percepciones que en su mayoría se obtuvo de acuerdo con cada tipo de socio.

\section{RESULTADOS}

\section{Resumen de respuestas de la percepción de los socios administradores:}

“... La participación ha disminuido drásticamente..." “...ya nos les interesa la agrupación...” “... Les pedimos su licencia de manejo para obligarlos a venir a las actividades de la organización..." "...cuando requerimos de la presencia de los operadores les quitamos su licencia para obligarlos a venir y a los que no vienen los sancionamos..." "... Son malagradecidos..." "... Ya no les interesa la agrupación a partir de que les pusimos nómina...en administraciones anteriores si participaban... porque eran participes del sindicato... no se les había dicho que ya no pertenecían a él... ..." “...creo que simplemente no les gusta los cambios... son gente sin estudios... de mente dura..." “... Pues es que antes se les pagaba diario... ahora con la nómina el tesorero se atrasa hasta dos o 3 semanas con el pago de su sueldo... es normal que lo pidan... ya lo trabajaron..." “... Se ha estado perdiendo presencia en actos de gobierno ... o cuando hay algún problema en la calle.... en las rutas... en paraderos... pues ya no contamos con el apoyo de los operadores para hacer presencia... ahora solo vamos socios administradores... pero no es el mismo efecto..."

\section{Resumen de respuestas de la percepción de los socios operadores:}

“... ya no nos invitan a los eventos de la organización..." "...antes nos festejaban el día del padre..." "...nos sancionan por todo..." "...ya no tenemos quien nos represente..." "....al parecer ya no tenemos sindicato..." "...creo que el presidente es el secretario general del sindicato" "...ya cambio todo..." "...ya no tenemos derecho a votar, solo ellos eligen..." "...ya ni nos preguntan nada... parece que ya no existimos..." "... somos los que trabajamos diario...." "...ellos se la pasen en las oficinas con sus aires acondicionados..." "...ya no se manchan las 
CAMBIOS QUE AFECTAN EL CLIMA ORGANIZACIONAL DE UNA EMPRESA DE TRANSPORTE.

manos..." "...ya se les olvido que eran operadores también..." “...nos maltratan como empleados, ni el sueldo pagan a tiempo...".

\section{CONCLUSIONES}

Debido a los cambios que se contemplan en la organización, tanto de manera legal, administrativa y social, se observa que las condiciones de identidad de los integrantes del que fuere el sindicato migro rápidamente hacia una posición totalmente desventajosa que las personas interpretan como una segregación.

Asimismo, todos tenían la misma posición social dentro de la empresa y como parte del inicio de la estructura, todos podían acceder a los mismos derechos y beneficios, siendo estos recortados, cambiados y suprimidos con el paso del tiempo, en consecuencia, vino un cambio en el clima organizacional.

Todos los cambios trajeron una serie de descontentos que los integrantes de la organización no terminan de aceptar, ya que aún siguen teniendo las obligaciones de sindicalizados; sin embargo, ya no tienen los derechos, lo que les causa una confusión que termina por ser concebida como un repudio por parte de su organización y que por ende ellos generan el mismo sentimiento hacia su empresa.

En los siguientes vínculos se toman notas de medios digitales en el que en diferentes estados de la república se dieron estos estallidos sociales posteriores a cambios similares que sufrieron los sindicatos a los que pertenecían y que hoy se identifican como empresas:

https://www.capitalqueretaro.com.mx/local/operadores-del-transporte-publico-de-queretarodetienen-labores-por-quejas-y-afectan-a-usuarios/

https://www.elimparcial.comsonora/hermosillo/El-miercoles-4-paro-parcial-en-HermosilloSindicato-del-Transporte-Urbano-20200301-0062.html

https://www.lajornadamaya.mx/2019-05-22/Alianza-de-Camioneros-inicia-paro-en-Yucatan https://www.elsoldetoluca.com.mx/local/por-aumento-de-pasaje-paro-laboral-de-choferes-delinea-ocho-de-noviembre-4654094.html

https://www.elsoldetlaxcala.com.mx/local/inician-transportistas-de-usu-paro-laboral-acusanexplotacion-de-trabajo-por-su-empresa-4119457.html

https://www.lja.mx/2020/03/en-aguascalientes-hay-deficit-de-choferes-de-transporte-publico/ MANZANERO-CHAN M. A., SABIDO-DOMÍNGUEZ T. DE J. 
CAMBIOS QUE AFECTAN EL CLIMA ORGANIZACIONAL DE UNA EMPRESA DE TRANSPORTE.

Como se puede observar, en los vínculos anteriores, las acciones en común que toman los integrantes de la organización, es el descontento generalizado que torna un ambiente negativo, se agrupan en pequeñas células con la intención de formar su propio sindicato y terminan por tener una actitud rebelde y poco conciliadora.

El resultado del clima organizacional, dada la nueva actitud que toman los operadores de la empresa, se ve obviamente dañado por que los operadores no se sienten comprometidos con los objetivos de la empresa, por el contrario, se perciben en el último escalón de la estructura administrativa y relegados de cualquier opinión o atención.

Al tener operadores descontentos, se afectan los resultados de la empresa, lo que hace que cambie también la conducta y la percepción de los socios administradores, haciéndose un círculo vicioso que termina por dañar a la empresa en general, limitándola de sus acciones y alejándola de sus objetivos, creando un estado de inestabilidad que podría finalizar dañando no solo el clima sino la cultura organizacional, lo que pondría en un estado de amenaza la existencia misma de la organización.

\section{REFERENCIAS BIBLIOGRÁFICAS}

Bote, A. (22 de mayo 2019). Alianza de Camioneros inicia paro en Yucatán. La jornada. Recuperado de https://www.lajornadamaya.mx/2019-05-22/Alianza-de-Camioneros-iniciaparo-en-Yucatan

Carmona Rojas, M. Y. (2016). Una lectura del transporte público concesionado en Ciudad de México desde la visión del orden urbano. Sociedad y Economía, (31), 71-94. Retrieved March 28, 2021, from http://www.scielo.org.co/scielo.php?script=sci_arttext\&pid=S1657$63572016000200004 \&$ lng=en\&tlng=es.

Domínguez Aguirre, L. R., Ramírez Campos, L. F. \& García Méndez, A. (2013). El clima laboral como un elemento del compromiso organizacional. Revista Nacional de Administración, 4(1), 59-70. https://doi.org/10.22458/rna.v4i1.533 
CAMBIOS QUE AFECTAN EL CLIMA ORGANIZACIONAL DE UNA EMPRESA DE TRANSPORTE.

Flores, A. (11 de marzo 2020). En Aguascalientes Hay déficit de choferes de transporte público. LJA.MX. Recuperado de https://www.lja.mx/2020/03/en-aguascalientes-hay-deficit-dechoferes-de-transporte-publico/

Galarza, A. O. (2015). Problemas teóricos del clima organizacional: Un estado de la cuestión. Revista de psicología, 12(1), 347-362.

González, G. (01 de marzo 2020). El miércoles 4 paro parcial en Hermosillo: Sindicato del Transporte Urbano. El imparcial. Recuperado de https://www.elimparcial.com sonora/hermosillo/El-miercoles-4-paro-parcial-en-Hermosillo-Sindicato-del-TransporteUrbano-20200301-0062.html

Hernández, I. (02 de marzo 2018). Qrobús detiene labores por quejas; paro parcial afecta a usuarios. Recuperado de https://www.capitalqueretaro.com.mx/local/operadores-deltransporte-publico-de-queretaro-detienen-labores-por-quejas-y-afectan-a-usuarios/

Hernández, R., Fernández, C. y Baptista. M. (2010). Metodología de la Investigación (5ª ed.). México: McGraw-Hill / Interamericana Editores S.A. de C.V.

Molina-Sabando, L., Briones-Véliz, Í. \& Arteaga-Coello, H. (2016). El comportamiento organizacional y su importancia para la administración de empresas. Dominio de las Ciencias, 2(4), 498-510. doi:http://dx.doi.org/10.23857/dc.v2i4.275

Pérez López, R. \& Landin Álvarez, J. M. (2019). Movilidad cotidiana, intermodalidad y uso de la bicicleta en dos áreas periféricas de la Zona Metropolitana del Valle de México. Cybergeo: European Journal of Geography.

Ramos, F. (3 de enero 2020). Por aumento de pasaje; paro laboral de choferes de línea Ocho de noviembre. El sol de Toluca. Recuperado de https://www.elsoldetoluca.com.mx/local/por-aumento-de-pasaje-paro-laboral-de-choferesde-linea-ocho-de-noviembre-4654094.html

Rivera, V. (2000). Llegando tarde al compromiso: La crisis del transporte en la Ciudad de México. México, D.F.: El Colegio de México. doi:10.2307/j.ctv3f8qbr

Salazar, J., Guerrero, J., Machado, Y. y Cañedo, R. (2009). Clima y cultura organizacional: dos componentes esenciales en la productividad laboral. ACIMED, 20(4), 67-75. Recuperado 
CAMBIOS QUE AFECTAN EL CLIMA ORGANIZACIONAL DE UNA EMPRESA DE TRANSPORTE.

de http://scielo.sld.cu/scielo.php?script=sci_arttext\&pid=S1024$94352009001000004 \& \operatorname{lng}=e s \&$ tlng=es

Schmalz, S. (2017). Los recursos de poder para la transformación sindical. Nueva sociedad, 272, 19-41.

Tlachi, E. (01 Septiembre 2019). Inician transportistas de USU paro laboral; acusan explotación de trabajo por su empresa. El Sol de Tlaxcala. Recuperado de https://www.elsoldetlaxcala.com.mx/local/inician-transportistas-de-usu-paro-laboralacusan-explotacion-de-trabajo-por-su-empresa-4119457.html 